\title{
Crystal surface reactivity analysis across scales: combined experimental and numerical insight
}

CORNELIUS FISCHER ${ }^{1}$, WOLF-ACHIM KAHL ${ }^{2}$, TAO YUAN $^{1}$, TILL BOLLERMANN ${ }^{1}$, WOLFGANG BACH ${ }^{2,3}$

${ }^{1}$ Helmholtz-Zentrum Dresden-Rossendorf (HZDR), Inst. für Ressourcenökologie, Abt. Reaktiver Transport, Leipzig, Germany

${ }^{2}$ Department of Geosciences, Univ. of Bremen

${ }^{3}$ MARUM Center for Marine Environmental Sciences, Univ. of Bremen, Germany

Dissolution rates of porous crystalline materials reflect competition between surface- versus transport-controlled mechanisms as a function of disequilibrium. As a result, reacting surfaces evolve over time showing a heterogeneous distribution of surface rates. The spatiotemporal heterogeneity of surface reaction rates is analyzed using the rate map and rate spectra concept. Here, we quantify the dissolution rate variability covering the $\mathrm{nm}$ - to $\mathrm{mm}$-scale of dissolving single crystal and polycrystalline calcite samples, using a combined approach of X-ray micro-computed tomography $(\mu-\mathrm{CT})$ and vertical scanning interferometry (VSI) [1]. Direct measurements of reaction rates of a crystal surface including corners and edges with high kink site density show the link between literature data collected on cleavage planes versus powder surfaces, i.e., the observation of an equivalent phenomenon of favored detachment at high kink density via distinctly different experimental strategies [2]. The rate map data underscore the concurrent and superimposing impact of surface- vs. fluid flow controlled rate portions. The impact of fluid flow on reactivity at the pore scale in the transport-controlled system is confirmed by reactive transport modeling. Current RTM uses transport control as the sole arbiter of differences in reactivity. As a general conclusion, numerical reactive transport concepts would benefit from the implementation of a reactivity term implementing the experimentally observed existence of multiple rate components, as exemplified in this study by an improved numerial approach.

[1] Kahl, W.-A.; Yuan, T.; Bollermann, T.; Bach, W.; Fischer, C., Crystal surface reactivity analysis using a combined approach of X-ray micro-computed tomography and vertical scanning interferometry. 2020, https://doi.org/10.2475/01.2020.03

[2] Bollermann, T.; Fischer, C., Temporal evolution of dissolution kinetics of polycrystalline calcite. Am. J. Sci. 2020, https://doi.org/10.2475/01.2020.04 